\title{
Molecular characterization of pathogenic Leptospira sp. in small mammals captured from the human leptospirosis suspected areas of Selangor state, Malaysia
}

\begin{abstract}
Leptospirosis is caused by the spirochetal bacterium Leptospira of which rodents are considered the most important reservoir. This study aims to determine and characterize virulent Leptospira species among rodents and small mammals found in human settlements and recreational spots within the Hulu Langat and Gombak districts of Selangor, Malaysia; regions that frequently report probable human leptospirosis cases. Molecular analysis revealed an overall Leptospira detection rate of $14.3 \%$ among the 266 small mammals captured, and the human settlements were found to have the highest number of isolates (15.1\%), followed by recreational sites $(14.5 \%)$. The molecular characterization conducted based on the lipL32, secY genes and MLST revealed that the strains belonged to four different species, including; Leptospira interrogans (29; 76.3\%; ST50, ST238, ST243), L. kirschneri (5; 13.15\%; ST110), L. borgpetersenii (3; 8\%; ST143) and L. weilii (1; 2.63\%; ST242). The study revealed genotypes of circulating strains among small mammals in Malaysia, which include Leptospira locus ST110 L. kirschneri, ST 50 L. interrogans, ST143 L. borgpetersenii and ST242 L. weilii. Among the small mammals studied, 17/105 (16.2\%) Rattus norvegicus, $7 / 59$ (11.9\%) of Rattus rattus, 5/24 (20.8\%) of Maxomys whiteheadi, 4/18 (22.2\%) of Sundamys muelleri, 2/22 (9\%), Tupaia gliss, 2/16 (12.5\%) Rattus tiomanicus and $1 / 4(25 \%)$ of Suncus murinus carried pathogenic leptospires. The data from the present study may imply that, in addition to rodents, other small mammals also serve as maintenance hosts for Leptospira. Hence, much remains unknown about Leptospira maintenance hosts, and there is need for further investigation to ascertain the prevailing serovars of pathogenic Leptospira in Malaysia. This will assist in the development of efficient diagnostic assays with improved microscopic agglutination test (MAT) panels, and in the implementation of suitable prevention and control measures.
\end{abstract}

Keyword: Leptospira; Leptospirosis; Malaysia; Rodents; Small mammals; L. interrogans; L. kirschneri; L. borgpetersenii; L. weilii; Recreational forest 